\title{
Die gesamtwirtschaftlichen Wirkungen der EU-Strukturpolitik in den Konvergenzregionen
}

Methodische Anmerkungen und empirische Ergebnisse für die Förderperiode

2007-2013

\section{The macro-economic impacts of the European Union structural policy in the convergence regions}

Methodical notes and empirical results for the period 2007-2013

\section{Kurzfassung}

Die Reform der europäischen Strukturpolitik in den späten 1980er Jahren stellte die Europäische Kommission und die nationale Politik und auch die Politikevaluation vor neue Aufgaben. Im Kontrast zu vielen anderen politischen Interventionen gehen die Maßnahmen der europäischen Strukturfonds explizit über eine nachfrageseitige Stabilisierung hinaus und sollen in erster Linie dazu beitragen, die angebotsseitigen Bedingungen in den unterstützten Volkswirtschaften zu verbessern. Die Förderung zielt darauf ab, durch eine Verbesserung der Produktionsbedingungen einen Beitrag zur Konvergenz der Einkommen innerhalb Europas zu leisten. Zur Analyse der Wirkungen sind eine Vielzahl von Methoden anwendbar, die sich hinsichtlich der volkswirtschaftlichen Reichweite der Wirkungen unterscheiden. In diesem Beitrag wird auf einige methodische Aspekte insbesondere zu den gesamtwirtschaftlichen Wirkungen eingegangen, und für die Förderperiode 2007-2013 werden einige ausgewählte Ergebnisse, die sich aus den HERMIN-Modellen ergeben, für die Empfängerländer dargestellt. Insgesamt zeigen sich signifikant positive Wirkungen für das Wachstum des Sozialprodukts wie auch der Beschäftigung, wobei es über die Empfängerregionen hinweg deutliche Unterschiede gibt, die auf die unterschiedlichen strukturellen Bedingungen in den Volkswirtschaften zurückgeführt werden können.

\begin{abstract}
The reform of the European structural policy in the late eighties confronted the European Commission and the national policy-makers with new tasks. In contrast to many other political interventions the measures of the European Structural Funds go out explicitly over short-term stabilisation and are supposed primarily to contribute to improve the supply-side conditions in the supported national economies. The promotion scheme has the target to improve of the production conditions and to contribute for the convergence of the incomes with in Europe. For the analysis of the effects are applicable a multiplicity of methods, which differ according to the economical range of the effects. In this contribution we deal with some methodical aspects in particular the overall economic effects and for the Structural Fund period 2007 to 2013. We present some selected results for the recipient countries, which come from the HERMIN models. Altogether significantly positive effects show up both for the growth of the national product and employment, whereby there are significant differences across the recipient countries, which can be traced backt to the different structural conditions within these economies.
\end{abstract}




\section{Einleitung}

Die Reform der europäischen Regional- und Strukturpolitik Ende der 1980er Jahre stellte sowohl die Europäische Union als auch die nationalen und regionalen wirtschaftspolitischen Akteure vor große Herausforderungen. Auf der einen Seite bestand die Aufgabe darin, nationale Programme zu entwickeln, die den Zielsetzungen der europäischen Strukturpolitik entsprachen, und zum anderen ergaben sich neue und weitreichende Anforderungen, um eine adäquate Wirkungsanalyse zu gewährleisten. Das Finanzvolumen der europäischen Strukturfonds stellte sich dabei nicht als das größte Problem dar, obwohl es mit etwa $1 \%$ bis $4 \%$ des nationalen BIP der Empfängerländer erheblich war. Eine Vielzahl mikroökonomischer Techniken stand zur Analyse der Wirkungen einzelner Projekte oder Maßnahmen zur Verfügung, wie die Kosten-Nutzen-Analyse, die Nutzwertanalyse oder ähnliche Verfahren. Auch auf makroökonomischer Ebene standen Methoden zur Beurteilung der (kurzfristigen) Wirkungen öffentlicher Ausgabenprogramme bereit. Sie sind ein Bestandteil der Forschung der quantitativen Makroökonomie seit den 1930er Jahren (Tinbergen 1939) und werden regelmäßig zur Abschätzung der nachfrageseitigen Wirkungen von Politikinterventionen eingesetzt. Das spezielle an den europäischen Strukturfonds ist aber, dass diese explizit darauf abzielen, eine fundamentale, strukturelle Erneuerung der Wirtschaftsstruktur der Empfängerländer zu unterstützen, um sie auf die Anforderungen vorzubereiten, die sich aus den veränderten Wettbewerbsbedingungen im Rahmen des europäischen Binnenmarkts ergeben. Dabei ergänzen die ausgabenintensiven Programme der EU-Strukturfonds diejenigen Politikinterventionen, die den ordnungspolitischen Rahmen betreffen.

Die Maßnahmen der EU-Strukturfonds bewegen sich deutlich über die gängigen nachfrageseitigen Stabilisierungspolitiken hinaus und versuchen den strukturellen Wandel, das langfristige Wachstum und die reale Konvergenz der Einkommen innerhalb der EU durch angebotsseitige Politikinterventionen zu forcieren. Auslöser für diesen Politikwechsel war die Sorge, dass die Beseitigung der nicht-tarifären Hemmnisse im Handel und die zunehmende Faktormobilität im Zuge des europäischen Binnenmarkts - ausgelöst durch die Änderung des ordnungspolitischen Rahmens - die Divergenzen zwischen reichen und armen Volkswirtschaften (sowohl in nationaler als auch in regionaler Hinsicht) noch intensivieren würde. Die Maßnahmen im Rahmen der EU-Strukturfonds sollten daher für die peripheren Volkswirtschaften die Bedingungen für die Ausnutzung von Handels- und Wohlfahrtsgewinnen verbessern und gleichzeitig die Risiken der Handelsliberalisierung minimieren oder reduzieren. Die Maßnahmen sind systematisch darauf gerichtet, durch verschiedene Investitionsprojekte in bestimmten EU-Mitgliedstaaten und Regionen den Strukturwandel voranzutreiben und die regionale Wettbewerbsfähigkeit zu stärken. Im Kern zielen die EU-Strukturfondsinterventionen deshalb darauf $a b$, die unterstützte Wirtschaft so zu verändern, dass sie auf Dauer im nationalen und internationalen Wettbewerb bestehen kann und Anschluss an das europäische Produktivitätsniveau findet.

In diesem Beitrag werden die erwarteten Wirkungen der EU-Strukturfonds auf die Regionen und Staaten für die Förderperiode 2007-2013 untersucht, die als sogenannte Konvergenzregionen (in der Förderperiode 2000-2006 als Ziel-1-Regionen bezeichnet) seitens der europäischen Strukturpolitik unterstützt werden. Diese Regionen, die durch ein Bruttoinlandsprodukt pro Kopf von weniger als $75 \%$ des EU-Durchschnitts gekennzeichnet sind, zählen zu den "armen“ Regionen der EU. ${ }^{1}$ Von den insgesamt für die Förderperiode 2007-2013 vorgesehenen EU-Finanzmitteln von $308,041 \mathrm{Mrd} . €$ (in Preisen von 2004 ) werden $81,54 \%$ für die Entwicklung der Konvergenzregionen verwendet. $^{2}$ Die übrigen Finanzmittel werden unter dem regionalen Wettbewerbs- und Beschäftigungsziel, das alle Regionen innerhalb der Union umfasst, die nicht zu den Konvergenzregionen gehören, und für das Ziel der europäischen territorialen Zusammenarbeit eingesetzt. Auf die erste Gruppe entfällt ein Anteil von 15,94 \% und auf die letztgenannte ein Anteil von 2,52\%.

Eine Wirkungsanalyse der EU-Strukturfonds kann sowohl aus gesamtwirtschaftlicher wie aus einzelwirtschaftlicher Perspektive vorgenommen werden. Es handelt sich hierbei um fundamental unterschiedliche Ansätze, wobei jeder Ansatz über spezifische Vor- und Nachteile verfügt. Zur Beurteilung einzelner Projekte oder Maßnahmen eignen sich mikroökonomisch ausgerichtete Verfahren (Bottom-up-Ansätze). Allerdings ist es nicht möglich, durch Addition der Wirkungen einzelner Projekte auf die gesamtwirtschaftliche Bedeutung einer Maßnahme oder des gesamten Programms zu schließen. Auf der anderen Seite ermöglichen es die gesamtwirtschaftlichen Ansätze (top-down), die Auswirkungen mit all ihren Wechselwirkungen in einer Volkswirtschaft auf die zentralen Zielvariablen Wachstum und Beschäftigung zu bestimmen; sie sind aber nicht in der Lage die Vorteilhaftigkeit einzelner Vorhaben zu bestimmen. Eine umfassende Evaluation der EU-Strukturfonds sollte beide Elemente enthalten, da sie sich wechselseitig positiv beeinflussen und zu einem Gesamtbild der Wirkungen zusammengesetzt werden können. Tabelle 1 fasst die Unterschiede der beiden Vorgehensweisen kurz zusammen. Auf der Projektebe- 
Tabelle 1

Unterschiede zwischen dem mikro- und makroökonomischen Evaluationsansatz

\begin{tabular}{|lll|}
\hline & Mikro (bottom-up) & Makro (top-down) \\
\hline Allgemeine Struktur & Informal, flexible, Nutzung subjektiver & Formal, komplex, objektiv, basierend auf \\
Disaggreationsniveau & Elemente & ökonomischen Theorien \\
Nutzung von Theorien & Hoch (einzelne Projekte) & Niedrig (Aggregate) \\
Modellkalibrierung & Schwach (Bewertungen) & Ausgeprägt (Makroökonomie) \\
Wirkungen & Bewertung & Ökonometrie \\
Behandlung von Externalitäten & Omplizit, Rangfolgen & Explizit, Quantitativ \\
\hline
\end{tabular}

Quelle: Bradley, Morgenroth, Mitze, Untiedt (2006)

ne wird in der Regel ohne Rückgriff auf ein formales Modell eine Beurteilung vorgenommen, die Struktur der Projektbeurteilungen ist informal und durch subjektive Elemente gekennzeichnet. Projekte werden im Allgemeinen in Rangfolgen gebracht und dann ausgewählt, mögliche Spillovereffekte werden kaum berücksichtigt. In Gegensatz zu dieser Vorgehensweise wird bei makroökonomischen Ansätzen eine formale Analysestruktur verwendet, die sich aus der ökonomischen Theorie ergibt. Zur Bestimmung der Wirkungen wird stark auf statistische und ökonometrische Methoden zurückgegriffen und die möglichen Externalitäten der Förderprogramme werden explizit berücksichtigt.

In der Evaluationspraxis sind die meisten einzelwirtschaftlichen Bewertungen zu den EU-Strukturfondsinterventionen - auch wenn es Standardtechniken zur mikroökonomischen Evaluation gibt - „Monitoringberichte “ und keine „Evaluationen" der eingesetzten Politiken. Erst in jüngster Zeit gibt es einige Versuche (Honohan 1997, Bradley et al. 2006), systematisch mikroökonomisch fundierte Strukturfondsbewertungen durchzuführen und im Rahmen eines kombinierten mikroökonomischen und gesamtwirtschaftlichen Ansatzes die Wirkungen zu analysieren.

Dieser Beitrag beschränkt sich auf die makroökonomische Perspektive. Dabei wird zunächst auf verschiedene Methoden und ihre Ergebnisse eingegangen und versucht, die Frage nach der Angemessenheit der eingesetzten Methoden zur Bewertung der EU-Strukturfonds zu beantworten. Anschließend wird im 3. Kapitel auf einen von den Autoren vertretenen makroökonomischen Modellansatz eingegangen, die so genannten HERMIN-Modelle, die im Rahmen der Bewertung der EU-Strukturfondsinterventionen breite Anwendung gefunden haben. Anschließend wird ein Überblick über mögliche Wirkungen der EU-Strukturfonds in der Förderperiode 2007-2013 gegeben. Zum Abschluss werden die Ergebnisse zusammengefasst und eini- ge Schlussfolgerungen für die weitere Evaluation der Strukturfonds gezogen.

\section{Möglichkeiten zur Modellierung der Wirkungen der europäischen Strukturfonds}

Die Analyse des Erfolgs oder der Wirksamkeit der Europäischen Förderpolitik kann auf verschiedenen Stufen erfolgen, die als Mikro- bzw. Meso- oder Makrountersuchungen der Förderpolitik bezeichnet werden können. Es ist hilfreich, die verschiedenen Ansätze kurz gegeneinander abzugrenzen und einen makroökonomischen Ansatz zu begründen. Auf der Ebene eines einzelnen Projekts ( $z$. B. Bau einer Straße, Errichtung eines Gewerbegebiets, Qualifizierung von Erwerbspersonen, Unterstützung von Forschung und Entwicklung) wird die Vorteilhaftigkeit in der Regel durch eine konventionelle Nutzen-Kosten-Analyse untersucht und die Verflechtung zur Gesamtwirtschaft vernachlässigt, was sich durch den geringen Mitteleinsatz des Einzelprojekts im Verhältnis zur Gesamtwirtschaft rechtfertigen lässt. Bewegt man sich auf der Aggregationsskala weiter nach oben, so kann danach gefragt werden, ob durch die Gesamtzahl der Projekte einer Politikmaßnahme das direkte Ziel (z.B. Verbesserung der Verkehrsinfrastruktur im Vergleich zu einer Referenzregion - z.B. Westdeutschland, Verbesserung der Arbeitsmarktchancen einer bestimmten Gruppe, Erhöhung des Forschungsoutputs der unterstützen Unternehmen) erreicht worden ist.

Da die mikroökonomischen und mesoökonomischen Untersuchungen Teile der Volkswirtschaft ausblenden und lediglich partielle Ergebnisse bezüglich der Wirkungen hervorbringen (können), ist es notwendig, da die Interventionen mit den Europäischen Strukturfonds mit Blick auf die angestrebten Ziele gesamtwirtschaftlich formuliert sind, die gesamtwirtschaftlichen Wirkungen zu untersuchen. Daher sind auch verschie- 
dene methodische Ansätze zur Messung der (gesamtwirtschaftlichen) Wirkungen der EU-Strukturfondsinterventionen entwickelt und eingesetzt worden. Diese umfassen: Input-Output-(IO-)Modelle, ökonometrische Eingleichungs- und ökonometrische Mehrgleichungsmodelle.

Beutel (1993, 2002) wendet ein IO-Modell zur Messung des Einflusses der Strukturfonds auf Makroregionen (Ostdeutschland und das italienische Mezzogiorno) und auf die nationalen Volkswirtschaften (Griechenland, Irland, Portugal und Spanien) an. Die Aufgabe von IO-Modellen besteht darin, die Lieferungs- und Leistungsverflechtungen von Gütern und Dienstleistungen zwischen den Produktionssektoren einer Volkswirtschaft und die Lieferungen an die Endnachfrage auszuweisen. Für den Förderzeitraum 2000-2006 kommt Beutel zu signifikanten kurzfristigen nachfrageseitigen Effekten für die untersuchten Volkswirtschaften. Es ist jedoch sehr schwierig, in den (statischen) IO-Modellrahmen angebotsseitige (neoklassische) Anpassungsmechanismen zu integrieren, so dass die eigentlich interessierenden langfristigen Veränderungen auf der Angebotsseite, die das zentrale Ziel der EU-Strukturfonds sind, nicht beurteilt werden können.

Ein weiterer Ansatz zur Modellierung geht auf Treyz (1993) zurück, der in jüngster Zeit um Elemente der Neuen Ökonomischen Geographie ergänzt worden ist (vgl. Fan/Treyz/Treyz 2000). Ausgangspunkt ist dabei ein stark räumlich disaggregiertes Modell, mit im Wesentlichen einem Einkommens-Ausgabenkonzept, das keine angebotsseitigen Anpassungsmechanismen enthält, die aus den Strukturfondsinterventionen resultieren. Das neuere Modell ist noch in einem experimentellen Stadium und scheinbar schwierig mit Blick auf die Strukturfondsinterventionen zu operationalisieren.

Daneben existieren Einzelgleichungsmodelle zur Evaluierung der Europäischen Strukturfonds, die vielfach auf Wachstumsregressionen vom "Barro-Typ" basieren. Hierbei wird, abgeleitet aus dem neoklassischen Wachstumsmodell, die durchschnittliche jährliche Wachstumsrate des Einkommens pro Kopf für einen längeren Zeitraum durch das Niveau des Einkommens pro Kopf zu Beginn des Zeitraums und einer Reihe von weiteren erklärenden Variablen wie der Investitionsquote, der Bevölkerungsentwicklung, dem Humanressourcenbestand, der Infrastrukturausstattung usw. erklärt. Die Strukturfondsinterventionen erscheinen als weitere erklärende Größe. Ein Beispiel ist Tondl (1999), die diesen Modellrahmen unter Verwendung von kombinierten Zeitreihen- und Querschnittsdaten für Regionen einsetzt. Ein ähnliches Modell verwenden auch Ederveen/de Groot/Nahuis (2002) und Eggert et al. (2007). Die Ergebnisse innerhalb dieses Modell- rahmens sind nicht eindeutig. Es werden sowohl positive als auch nicht signifikante Wirkungen der Strukturfonds ermittelt. Mit diesem Modellansatz sind aber erhebliche methodische Probleme verbunden, die es fraglich erscheinen lassen, ob er hilfreich sein kann, um die Effekte der EU-Strukturfonds zu ermitteln. ${ }^{3}$

Vollständig spezifizierte gesamtwirtschaftliche Modelle wurden von Bradley/Whelan/Wright (1995), Roeger/ in't Veld (1996), Bradley/Morgenroth/Untiedt (2003) und Bradley/Untiedt (2007) vorgelegt. Der wesentliche Vorteil dieser modellbasierten Evaluationen besteht darin, den Einfluss der Interventionen im Vergleich zu einer Situation zu evaluieren, für die angenommen wird, dass es keine Politikintervention unter sonst gleichen Bedingungen gegeben hätte und zwischen den kurz- und langfristigen Wirkungen der Interventionen unterschieden werden kann. Natürlich spielen auch in diesen Modellen die theoretischen Grundlagen eine wesentliche Rolle bei der Bestimmung der Wirkungen der Interventionen. QUEST (Roeger/in't Veld 1996), ein makroökonomisches Mehr-Länder-Modell der Europäischen Kommission, wurde entwickelt, um die Volkswirtschaften der „alten“ EU-Mitgliedstaaten und ihre Interaktion mit dem Rest der Welt, insbesondere den USA und Japan zu analysiseren. Im Modellansatz stehen rationale Erwartungen im Zentrum der Analyse. Dieses führt im QUEST-Modell dazu, dass der enthaltene Crowding-out-Mechanismus zu einem geringeren Einfluss der Strukturfonds auf die kurzfristige Entwicklung der untersuchten Volkswirtschaft führt, während sich in der langen Frist signifikante positive Wirkungen auf Wachstum, aber nicht auf die Beschäftigungsentwicklung einstellen.

Der HERMIN-Modellrahmen (Bradley 1995, Bradley/ Untiedt 2007a) wurde ursprünglich zur Modellierung der Volkswirtschaften der Kohäsionsländer Irland, Portugal, Spanien und Griechenland entwickelt. Methodisch handelt es sich um ein neo-keynesianisches Modell, erweitert um neo-klassische Elemente mit adaptiven Erwartungen im Gegensatz zu den rationalen Erwartungen des QUEST-Modells. Explizit wird zwischen den kurzfristigen Keynesschen Effekten und den langfristigen angebotsseitigen Effekten unterschieden. Mittlerweile besteht das System aus 17 makroökonomischen Modellen für sämtliche Staaten, die unter dem Ziel „Konvergenz“ gefördert werden, und umfasst somit neben den „alten“ Kohäsionsländern auch sämtliche neuen Mitgliedstaaten und die Makroregionen Ostdeutschland und Italiens Mezzogiorno. Die HERMINModelle zeigen signifikante Keynessche Effekte in der kurzen Frist und positive langfristige Effekte der EUStrukturfonds sowohl für das Wachstum als auch die Beschäftigung in den unterstützen Volkswirtschaften. 
Die langfristigen Niveaueffekte auf das BIP sind in etwa gleich denen von QUEST. Der wesentliche Unterschied liegt aber bei den Beschäftigungswirkungen und ist Gegenstand einer wissenschaftlichen Diskussion (vgl. Bradley/Untiedt 2007b). Aber auch die HERMIN-Modelle weisen Schwachpunkte auf. Zum Beispiel sind die Evaluationsergebnisse abhängig von den gewählten Elastizitäten der langfristigen Einflussgrößen. Eine Erhöhung der Elastizitäten führt zu einer Erhöhung der Wirkungen der Strukturfonds, so dass im Allgemeinen Sensitivitätsanalysen bezüglich der Robustheit der Resultate ausgeführt werden müssen. Eine weitere mögliche Schwäche besteht in dem hohen sektoralen Abstraktionsgrad, obwohl mit einer sektoralen Untergliederung in fünf Sektoren (Landwirtschaft, Industrie, Dienstleistungen, Bau und Staat) bereits eine gewisse sektorale Disaggregation vorliegt. Das QUEST-Modell hingegen ist ein Einsektorenmodell und kann folglich keine Aussage zu den Wirkungen der EU-Strukturfonds auf die sektorale Entwicklung der unterstützen Volkswirtschaft leisten.

\section{HERMIN-Modelle zur Analyse der Wirkungen der EU-Strukturfonds ${ }^{4}$}

Die Notwendigkeit, die strukturverändernden Effekte der EU-Strukturfondsinterventionen $\mathrm{zu}$ analysieren, verbindet den Evaluationsprozess eng mit der aktuellen ökonomischen Forschung und benötigt ein ständiges Reflektieren der neuesten Erkenntnisse aus den Bereichen der Wachstumstheorie und der Analyse der langfristigen Wirkungen von Infrastruktur- und Humankapitalmaßnahmen auf die wirtschaftliche Entwicklung der unterstützten Regionen. Eine wichtige Eigenschaft, die eine auf den oben genannten Faktoren fußende makroökonomische Evaluation der Wirtschaft haben muss, ist die notwendige Transparenz des Vorgehens, um anschaulich die Wirkungszusammenhänge zu verdeutlichen, mit denen die EU-Strukturfonds das Wachstums- und Kohäsionsziel erreichen können.

Dabei ist zu beachten, dass die Interventionen der EUStrukturfonds die unterstützen Konvergenzregionen durch einen Mix aus Nachfrage- und Angebotseffekten beeinflussen. Die nachfrageseitigen Wirkungen entstehen während der Implementationsphase, wenn die Finanzmittel eingesetzt werden. Nach Abschluss der Förderperiode entfällt der nachfrageseitige Impuls und es wirken, sofern die Finanzmittel sinnvoll eingesetzt wurden, die aus der Verbesserung der angebotsseitigen Faktoren resultierenden Einflüsse fort. Wie bereits angesprochen sind die kurzfristigen keynesschen Nachfrageeffekte lediglich ein Nebenaspekt der Förderung und tragen nur zur kurzfristigen Stabilisierung der unterstützten Wirtschaft bei.
Die langfristigen Wirkungen der EU-Strukturfondsinterventionen stehen hingegen im Mittelpunkt des Interesses und ergeben sich über die Angebotsseite. Sie verändern die Produktionsbedingungen und -kosten und haben damit einen Einfluss auf den regionalen Anteil an der „Weltproduktion“, der durch einen Kostenwettbewerb bestimmt wird. So führt die Förderung der privaten Investitionstätigkeit zu einer Reduktion der Kapitalnutzungskosten und zu erhöhten Investitionen. Darüber hinaus müssen neben einem konkurrenzfähigen Niveau der Produktions- und Arbeitskosten weitere Standortfaktoren wie eine entsprechend qualifizierte Arbeitnehmerschaft und ein angemessenes Niveau an physischer Infrastruktur berücksichtigt werden. Beides wird in HERMIN erfasst, wie im Folgenden gezeigt werden soll.

Kurzfristige (Keynessche) Nachfrageeffekte entstehen durch die Erhöhung der (öffentlichen) Ausgaben und der privaten Einkommen. Durch die im HERMIN-Modell abgebildeten Multiplikatoreffekte wird der Impuls im volkswirtschaftlichen System weitergeleitet, was zu einem Wachstum maßgeblicher Aggregate (z. B. Investitionsvolumen, privater Konsum etc.) führt, wodurch sich wiederum das Gesamteinkommen erhöht. Im Rahmen der Strukturfondsförderung werden diese Mechanismen aufgrund ihres temporären Charakters jedoch nur als Nebeneffekte erfasst. Der Fokus der Förderung liegt auf der Verbesserung des langfristigen Potenzials der Angebotsseite. Diese Angebotseffekte entstehen durch

(1) die Verbesserung der physischen Infrastruktur als Inputfaktor der Produktionsaktivität im privaten Sektor,

(2) die Verbesserung des Humankapitals durch Investitionen in Bildung und Ausbildung als Inputfaktor der Produktionsaktivität im privaten Sektor und

(3) die Investitionsförderung im privaten Sektor zur Stimulierung von Investitionen und zur Erhöhung der Produktionseffizienz. Damit kann die Faktorproduktivität gesteigert und es können die sektoralen Produktions- und Kapitalkosten gesenkt werden.

In Abbildung 1 wird der Wirkungsmechanismus von Investitionen in die Infrastruktur auf das mittelfristige Ziel der EU-Strukturfonds aufgezeigt, wie durch die Verbesserung der Infrastruktur die allgemeine Wettbewerbsfähigkeit der Regionen verbessert werden kann. Investitionen in die Infrastruktur führen zu einer Reduktion der Produktionskosten (durch Senkung der Transportkosten), die Aufhebung bzw. Minderung von Produktionsrestriktionen und -engpässen (durch sinkende Lagerbestände) und eine Stärkung der wirtschaftlichen Rahmenbedingungen (z.B. durch ein 
Abbildung 1

Wirkungen von Investitionen in die Infrastruktur

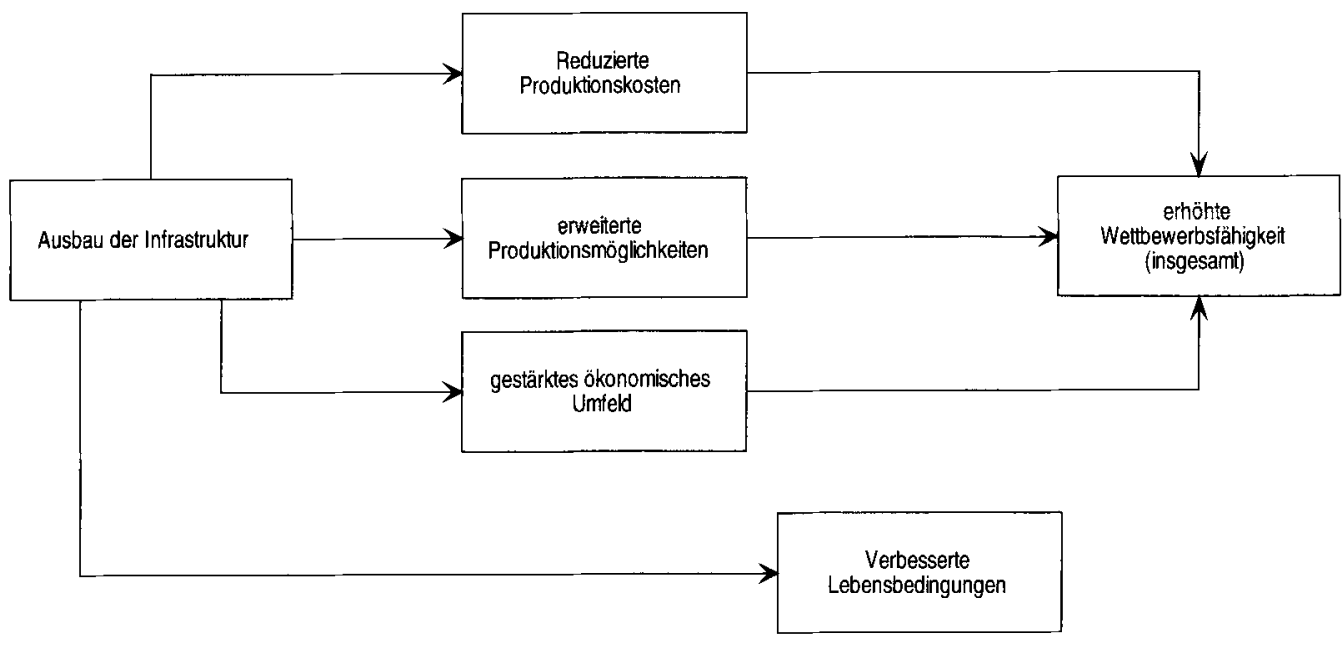

Wachstum der Nachfrage, vereinfachter Zugang zu Arbeitskräften etc.). Alle drei Faktoren wirken hin auf eine Verbesserung der allgemeinen Wettbewerbsvorteile der geförderten Region.

In welcher Form die gestiegenen Wettbewerbsvorteile der unterstützten Region zu einer nachhaltigen Verbesserung der wirtschaftlichen Leistung beitragen, wird in Abbildung 2 dargestellt. Auf der einen Seite wirken Wettbewerbsvorteile durch Faktormobilitäten auf Basis von relativen Kostenvorteilen, bei denen die Modellstrukturen weiterhin als fixiert bzw. exogen angenommen werden können. ${ }^{5}$ Auf der anderen Seite können die Mechanismen durch einen komplexen Wirkungsablauf die ökonomische Basis der Region durch Spezialisierungs- und Diversifikationseffekte stärken. Während die zuletzt genannten Wirkungsmechanismen relativ einfach in kleine, aus wenigen Gleichungen bestehende Wachstumsmodelle integriert werden können, ist die Implementation dieser Mechanismen in größere Makromodelle schwierig. Dennoch sind diese Wirkungsketten innerhalb der HERMIN-Modelle ein wichtiger Einflusskanal der EU-Strukturfonds und werden adäquat modelliert. Die oben genannten Mechanismen gelten dabei analog auch für die Verbesserung der Qualität des Humankapitals oder die Stärkung von Forschung und Entwicklung in den unterstützen Volkswirtschaften. Der zweite Wirkungsmechanismus wird im Folgenden auch als „endogene“ Entwicklung bezeichnet, da anders als bei den exogenen Prozessen die Modellstrukturen selbst (z.B. die Parameter der Produktionsfunktion) durch politische Interventionen geändert werden können.

Die obige Aufzählung der Angebotseffekte verdeutlicht, dass Politikinterventionen im Rahmen der EU-Strukturfonds insbesondere darauf ausgerichtet sind, das vorhandene Niveau an physischer Infrastruktur und Humankapital zu erhöhen und darüber hinaus den privaten Kapitalstock verbessern. Die Bereitstellung einer verbesserten und erweiterten Infrastruktur, die Erhöhung des Qualifikationsniveaus der Arbeitskräfte und die Bereitstellung von Investitionszuschüssen an private Unternehmen sind Mechanismen der Förderung zur Steigerung der Produktionshöhe, der Produktivität und der (Kosten-)Wettbewerbsfähigkeit der regionalen Volkswirtschaft. So können Firmen einerseits von zusätzlichen, produktiven Faktoren profitieren, die für sie keine Kosten darstellen (z.B. bei der Qualifizierung der Arbeitskräfte, der Bereitstellung von physischer Infrastruktur etc.) oder andererseits die Kosten bzw. relativen Preise für notwendige Inputfaktoren der Unternehmen reduzieren (z.B. durch Investitionszuschüsse). In beiden Fällen entstehen durch die EU-Strukturfonds positive Externalitäten für die private Wirtschaft.

In aktuellen Beiträgen zur „neuen“ Wachstumstheorie wird vermehrt auf die Bedeutung von Spillovereffekten und Externalitäten von öffentlichen Investitionsprogrammen hingewiesen, insbesondere im Bereich der physischen Infrastruktur und dem Humankapital (für eine Übersicht vgl. Bradley/Untiedt 2007a, de la Fuente 2006). Darüber hinaus werden in der Literatur positive Wachstumseffekte durch die direkte Förderung von Forschung und Entwicklung (FuE) diskutiert (vgl. Congressional Budget Office 2005). Letzte sind insbesondere dann zu beobachten, wenn die Förderprogramme den Charakter eines öffentlichen Gutes haben, d.h. Forschungsergebnisse durch Unternehmen nicht vollständig internalisiert werden, sondern auch unternehmensextern adaptiert werden (können).

Dabei können zwei wesentliche Externalitäten in Verbindung mit den angebotsseitigen Wirkungen von ad- 
Abbildung 2

Langfristige Wirkungen von Investitionen in die Infrastruktur

(1) Exogene Prozesse

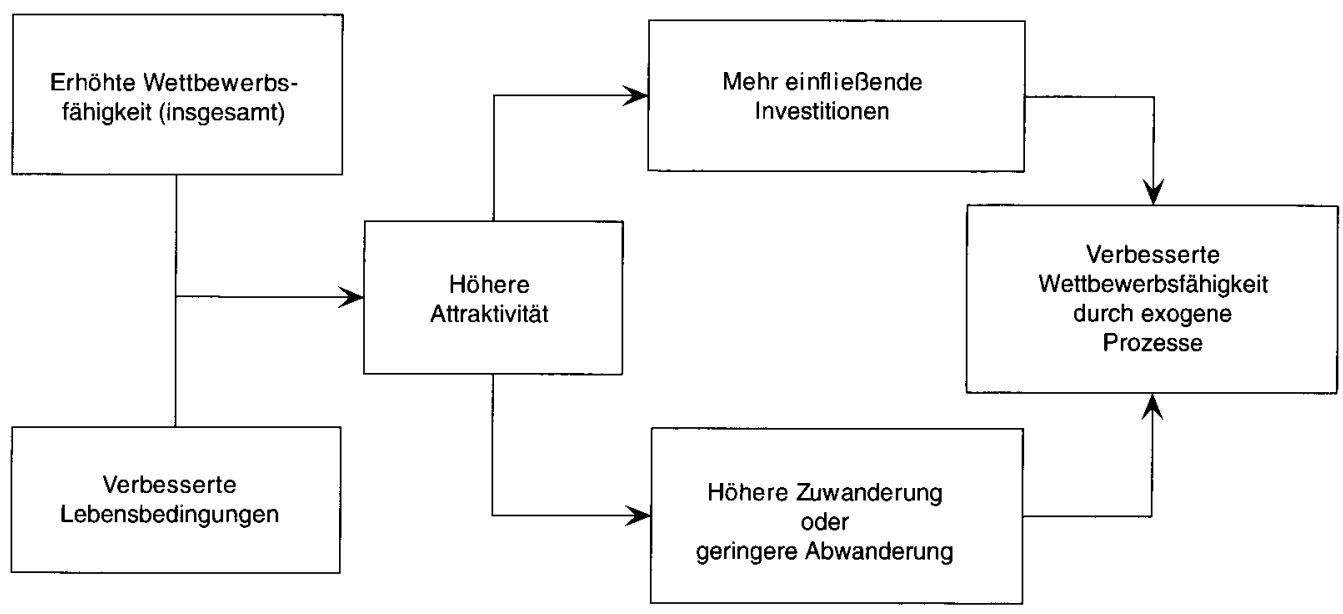

(2) Endogene Prozesse

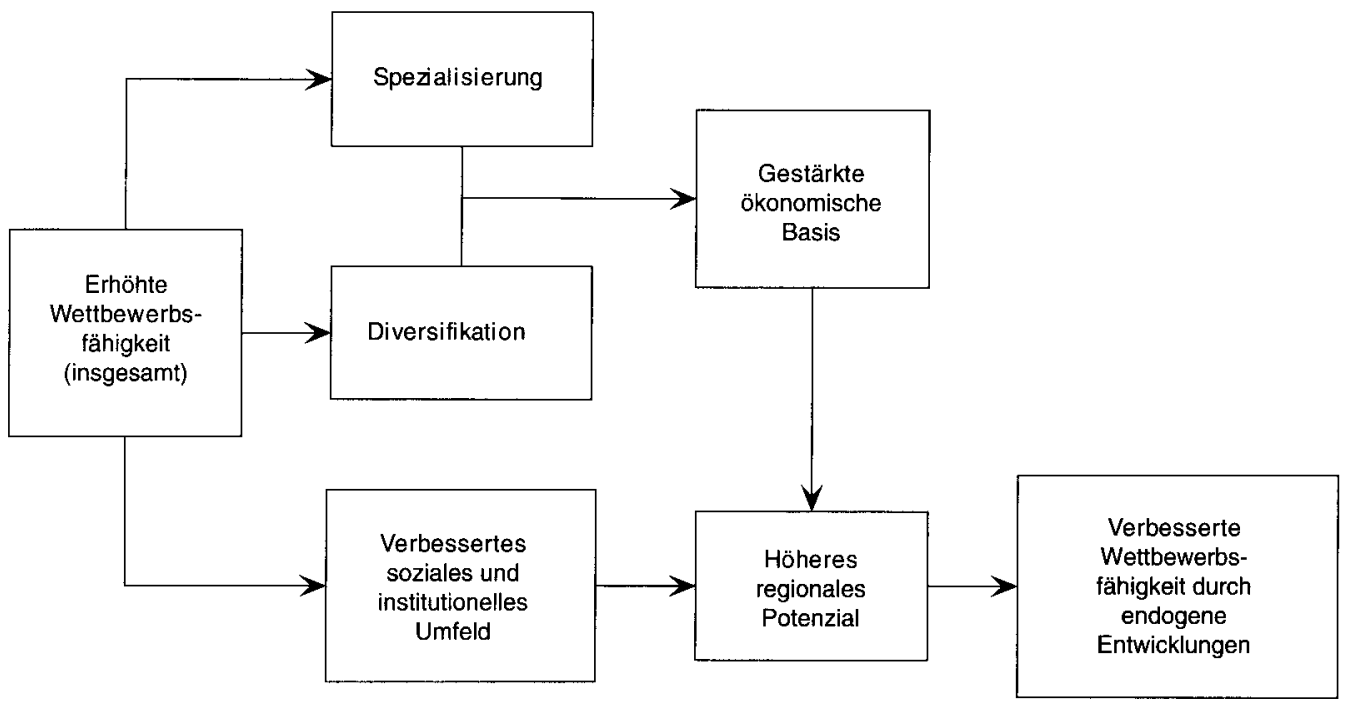

äquat ausgestalteten Investitions- und Bildungsinitiativen gesehen werden:

Die erste Externalität verbessert direkt über Investitionen in die physische Infrastruktur und die Qualifizierung der Arbeitskräfte die Produktion. Der Mechanismus der Outputsteigerung durch Verbesserung der Produktionsinputs wird durch das Attrahieren von ausländischen Direktinvestitionen oder die Verbesserung der Wettbewerbsfähigkeit realisiert. Diese direkt wirkende Externalität wird auch als Produktionsexternalität bezeichnet.

Die zweite Externalität wirkt über die Verbesserung der Faktorproduktivitäten als Resultat der Investitionsförderung durch die EU-Strukturfonds. Sie wird im Folgenden deshalb auch als Faktorproduktivitäts- externalität bezeichnet. Bei der Steigerung der Produktivität muss aber der Nebeneffekt beachtet werden, dass bei gegebenem Output die Beschäftigung sinkt und es zum sogenannten Jobless-growth-Phänomen kommen kann. Die Faktorproduktivitätsexternalität muss somit als ein zweischneidiges Schwert gesehen werden: Auf der einen Seite erhöht sie die Produktivität und Wettbewerbsfähigkeit im Produzierenden Gewerbe und Dienstleistungssektor, andererseits reduziert sie bei gegebenem Output die Arbeitsnachfrage. Dies trifft insbesondere für Volkswirtschaften zu, die bereits über hohe (versteckte) Arbeitslosenraten verfügen. Andererseits sollte beachtet werden, dass durch die Verbesserung der Produktivität das reale Einkommen steigt, was wiederum über Multiplikatoreffekte positive Impulse für die Entwicklung der Volkswirtschaft freisetzt. 
Während der Implementationsphase werden jährlich Investitionen getätigt. Ihr kumulativer Einfluss zeigt sich aber erst über das langfristige Ansteigen der Kapitalstöcke für Infrastruktur und Humankapital in der unterstützten Volkswirtschaft. Beispielsweise steigt mit Investitionen in die Verkehrsinfrastruktur die Qualität der Straßen. Wenn die Straßen miteinander verbunden sind, steigt auch die „Effektivität" des Infrastrukturbestands. Ähnlich lässt sich für den Bestand an Humankapital oder an Forschungskapazitäten argumentieren. Es sind generell diese Effekte, die Spillovereffekte generieren können und zu einer erhöhten Produktion und höherer Produktivität führen, damit den Konvergenzprozess treiben können.

Es ist erstrebenswert, die Parameter für die Elastizitäten der Kapitalstöcke und damit ihren Einfluss auf das Sozialprodukt für jedes Empfängerland von EUStrukturfondsmitteln separat zu bestimmen, sei es auf der Basis eigener Untersuchungen oder veröffentlichter Forschungsergebnisse. Jedoch gibt es weder für die neuen Mitgliedstaaten noch für Ostdeutschland und für die „alten“ Kohäsionsländer mit Ausnahme von Spanien ausreichend Forschungsarbeiten, die die Spilloverelastizitäten schätzen. International ist der Kenntnisstand bezüglich der öffentlichen Infrastruktur besser als für das Humankapital, und gesamtwirtschaftliche Spillovereffekte für Forschung und Entwicklung sind selten. Hier besteht weiter dringender Forschungsbedarf; die aktuelle empirische Wachstumsforschung versucht bereits mehr Licht in das Dunkel zu bringen. Neben den individuellen Spillovereffekten der einzelnen Interventionskategorien stellt sich auch die Frage nach dem optimalen Mix der Investitionen in die Infrastruktur und das Humankapital. Dieser Zusammenhang wird aber erst in jüngster Zeit in der Wachstumstheorie thematisiert, und über eine optimale Allokation der Ressourcen kann im Zusammenhang mit den EUStrukturfondsinterventionen wenig gesagt werden. Die Wahl der Zusammensetzung eines Programms, d.h. der individuellen Maßnahmen (vgl. Aghion/Howitt 2005) nach den verschiedenen Komponenten, ist mehr eine Kunst als wissenschaftlich begründbares Handeln. In den HERMIN-Modellen werden die Spilloverelastizitäten aufgrund der in der internationalen Literatur veröffentlichten Schätzwerte kalibriert; die Sensitivität der Ergebnisse wird über alternative Szenarien untersucht. Grundsätzlich werden konservative Schätzwerte für die Modellierung und zur Abschätzung der Wirkungen zugrunde gelegt.

Das Dilemma der gesamtwirtschaftlichen Analyse der Wirkungen der EU-Strukturfonds besteht darin, dass bei einer Ex-ante-Untersuchung der Auswirkungen nicht bekannt ist, wie gut oder schlecht die Opera- tionellen Programme auf die Notwendigkeiten und Bedürfnisse des Empfängerlandes hin ausgerichtet sind. Und weiterhin ist kaum abschätzbar, wie die Programme letztlich implementiert werden. Im besten Fall kann ein spezifisches Strukturfondsprogramm aufgrund der vorliegenden Dokumente und der Begründungen für spezifische Interventionen beurteilt werden. Weitere Informationsquellen zur Beurteilung der geplanten Interventionen können die ökonomische Theorie, eine detaillierte Kenntnis der untersuchten Ökonomie oder anderer den Strukturfonds ähnlicher Programme sein, die in der Vergangenheit in dieser Ökonomie implementiert wurden.

\section{4 Überblick zu einigen makroökonomischen Strukturfondswirkungen}

Im Folgenden werden die Ergebnisse der Ex-anteAnalyse der für den Zeitraum 2007-13 vorgesehenen Interventionen mit den Europäischen Strukturfonds vorgestellt. Für die einzelnen Empfängerregionen ist der finanzielle Impuls unterschiedlich hoch. Die finanziellen Impulse aufgrund der EU-Strukturfonds (einschließlich der nationalen Kofinanzierung) liegen in den neuen Mitgliedstaaten zwischen 5 und $6 \%$ des jährlichen realen BIP, für Griechenland, Portugal und das Mezzogiorno liegt der Wert um $2 \%$, für Ostdeutschland bei knapp $1 \%$ und für Spanien bei $0,7 \%$.

Bevor die Ergebnisse vorgestellt werden können, sind einige wichtige Entscheidungen zu treffen:

- Die in administrativen Kategorien formulierten Operationellen Programme der einzelnen Staaten müssen in Kategorien überführt werden, die für eine ökonomische Analyse zugänglich sind: Die einzelnen Maßnahmen werden den ökonomischen Kategorien Investitionen in die physische Infrastruktur, in die Humanressourcen und in private Investitionen zugeordnet.

- Die Definition einer geeigneten Benchmark-Projektion für den Fall der Abwesenheit von Strukturfondsinterventionen ist durchzuführen, aber unter Berücksichtigung der Wirkungen anderer Politiken, wie dem Gemeinsamen Markt, der europäischen Währungsunion, den Regelungen der WTO usw.

- Vorzunehmen sind eine Analyse der üblichen Keynesschen Effekte der EU-Strukturfondsprogramme auf die gesamtwirtschaftliche Nachfrage und die öffentlichen Finanzen und eine Quantifizierung der langfristigen angebotsseitigen Effekte der Strukturfondsprogramme.

- Zur Analyse der langfristigen Effekte sind die Spilloverelastizitäten für die Länder zu bestimmen. 
- Schließlich ist eine Quantifizierung der gesamten Wirkungen der Strukturfondsinterventionen als Abweichung von der Benchmarklösung zu ermitteln.

Auf jeder Stufe sind komplexe Entscheidungen zu treffen: Diese betreffen beispielsweise die Definition des Benchmarkszenarios. Dabei muss definiert werden, ob im Basisszenario die Strukturfonds gänzlich entfallen, zum Teil oder vollständig durch nationale Programme ersetzt werden usw. Bei den weiter unten aufgeführten Ergebnissen wurde unterstellt, dass die EU-Strukturfondsmittel und die nationale Kofinanzierung zusätzlich in den Wirtschaftskreislauf der unterstützten Volkswirtschaft einfließen. Private Kofinanzierungen werden hingegen nicht berücksichtigt.

Ohne genaue Kenntnis der Operationellen Programme (OP) und ihrer Ausgestaltung in den einzelnen Empfängerländern wird weiterhin angenommen, dass die OPs über die verschiedenen Empfängerländer eine identische Effektivität in der Umsetzung und damit potenziell gleiche Wirkungen aufweisen. Auf die Modellstruktur übertragen bedeutet dies, dass alle Modelle identische Spilloverelastizitäten aufweisen, die im Wesentlichen die langfristigen Effekte der Strukturfonds determinieren. Im Ergebnis sind dann die gemessenen Unterschiede in der Performance nicht mehr auf Unterschiede in der Wirkungsweise der EU-Strukturfonds in den Ländern zurückzuführen, sondern im Wesentlichen das Resultat der zugrunde liegenden makroökonomischen HERMIN-Modelle, die ein Abbild der untersuchten Volkswirtschaft sind. Die untersuchten Volkswirtschaften besitzen unterschiedliche Eigenschaften hinsichtlich des mikro- und makroökonomischen Ordnungsrahmens, der sektoralen Zusammensetzung, des Grades an außenwirtschaftlicher Offenheit etc., die sich dann in unterschiedlichen $\mathrm{Pa}$ rameterschätzwerten der strukturellen Gleichungen der HERMIN-Modelle niederschlagen und auf die Effizienz und Effektivität der EU-Strukturfonds Einfluss nehmen.

Während der Implementierungsphase, d.h. in der Periode von 2007 bis 2013, wenn die EU-Strukturfondsmittel verausgabt werden und die Kapitalstöcke an Infrastruktur, Humankapital und betrieblichem Anlagevermögen aufgebaut werden, kommt es temporär $\mathrm{zu}$ einem Anstieg der Wachstumsrate des BIP über die Wachstumsrate in der Baseline. Ist die Implementierungsphase für die aktuelle Förderperiode vorüber, verbleiben erhöhte Kapitalbestände. Da der nachfrageseitige Impuls entfällt, kehrt die Wachstumsrate des BIP auf das Ausgangsniveau zurück. Im Ergebnis haben die Strukturfondsinterventionen somit einen Niveaueffekt bezogen auf die relevanten Variablen, wie z. B. das BIP.
Die langfristige Wachstumsrate des BIP wird durch die EU-Strukturfonds nicht berührt.

Zentral für die europäischen Strukturfonds ist das Ziel, einen Beitrag zur realwirtschaftlichen Konvergenz der Einkommen zu leisten. Die vorübergehenden transitorischen Effekte während der Implementationsphase 2007-2013 sind dabei insgesamt weniger interessant als die langfristigen Effekte. In den durchgeführten Simulationen wird die Implementationsphase abrupt im Jahr 2013 gestoppt, es wird kein Nachfolgeprogramm aufgelegt, die Modelle werden aber mit den veränderten Kapitalstöcken für die Infrastruktur, das Humankapital und den betrieblichen Kapitalstock bis zum Jahr 2020 weiter simuliert. Durch einen Vergleich zwischen dem Basisszenario, der Entwicklung der betrachteten Volkswirtschaft ohne EU-Strukturfonds und der Modelllösung unter Einsatz der EU-Strukturfonds werden somit die langfristigen Effekte der Strukturfondsförderung sichtbar.

Die langfristigen Wirkungen auf das BIP in den Empfängerländern sind in Tabelle 2 für das Jahr 2020 aufgeführt. Die ausgewiesenen Werte entsprechen dem prozentualen Niveauunterschied im BIP im Vergleich zur Basislösung. Für die Tschechische Republik wird ein Unterschied im Niveau von 4,4 \% gegenüber der Basislösung für das Jahr 2020 ausgewiesen. In allen anderen Ländern sind die langfristigen Unterschiede geringer und betragen für Ostdeutschland gerade mal $0,15 \%$. Der absolute Unterschied im Niveau des BIP im Jahr 2020 zeigt, das langfristig positive Effekte seitens der

Tabelle 2

Niveauunterschied im BIP in \% im Jahr 2020 für die Förderperiode 2007-2013

\begin{tabular}{|lc|}
\hline & Differenz in \% \\
\hline Tschechische Republik & 4,40 \\
Ungarn & 4,10 \\
Estland & 3,70 \\
Polen & 2,70 \\
Slowenien & 2,10 \\
Rumänien & 1,70 \\
Portugal & 1,70 \\
Lettland & 1,40 \\
Italiens Mezzogiorno & 0,70 \\
Spanien & 0,30 \\
Griechenland & 0,30 \\
Ostdeutschland & 0,15 \\
\hline
\end{tabular}

Quelle: Bradley et al. 2004, S. $61 \mathrm{ff}$. 
EU-Strukturfonds verbleiben. Ihre Höhe ist aber auch eine Funktion des jährlichen Impulses während der Implementationsphase. Länder mit einem hohen Anteil der EU-Strukturfonds am BIP weisen langfristig höhere Werte auf als solche Länder, die einen geringeren Anteil haben. Auf diesen Punkt wird weiter unten detaillierter eingegangen. Wichtig ist es festzuhalten, dass der Einfluss der EU-Strukturfonds auf die realwirtschaftliche Konvergenz positiv ist, aber die EU-Strukturfonds den Konvergenzprozess nicht dominieren. Beispielsweise liegt in den neuen Mitgliedstaaten das Niveau der Einkommen pro Kopf in Relation zur EU-15 zwischen $30 \%$ für Rumänien und $80 \%$ für die Tschechische Republik. Die EU-Strukturfonds würden unter sonst gleichen Bedingungen dafür sorgen, dass die relativen Einkommen pro Kopf in Bulgarien auf 31,7 \% in Rumänien und $84,4 \%$ in der Tschechischen Republik ansteigen.

Die prozentuale Differenz zwischen der Baseline und der Modellösung unter Einsatz der EU-Strukturfonds in Tabelle 2 vernachlässigt den Impuls, den die unterstützte Volkswirtschaft erfährt. Volkswirtschaften, die einen hohen Impuls erfahren, zeigen auch große Unterschiede im Niveau des BIP. Ein geeignetes Maß, das einen Vergleich über die Regionen ermöglicht und den finanziellen Impuls berücksichtigt, muss das Ergebnis der Förderung zum Impuls in Relation setzen. Für diesen Zweck wurde der "kumulierte" StrukturfondsMultiplikator (SF-Multiplikator) zum Zeitpunkt j entwickelt. Dieser setzt die kumulierte jährliche Differenz zwischen der Modelllösung mit Strukturfonds und der Basislösung ins Verhältnis zu dem kumulierten Impuls der Implementationsphase. Ein kumulierter SF-Multiplikator von 1 bedeutet, dass die Wirkung gleich dem Impuls ist und keine langfristigen Effekte existieren. Ein SF-Multiplikator kleiner 1 zeigt an, dass die gesamten realwirtschaftlichen Effekte geringer sind als der Impuls. Dies kann sich ergeben, wenn Crowding-outEffekte dominieren oder bedingt durch die Struktur der unterstützten Wirtschaft die Vorteile nicht genutzt werden, die sich aus der Verbesserung der angebotsseitigen Bedingungen ergeben. Je größer der kumulierte SF-Multiplikator ist, umso effizienter sind die Strukturfondsmittel eingesetzt. Der kumulierte Strukturfonds-Multiplikator ist definiert als:

Kumulierter SF Multiplikator ${ }_{j}=$

$$
\frac{\sum_{t=2007}^{j}\left(\mathrm{BII}_{t}^{\text {mit Strukuturonds }}-\mathrm{BIP}_{t}^{\text {Baseline }}\right) / \mathrm{BIP}_{t}^{\text {Bascline * }} 100}{\sum_{t=2007}^{j} \mathrm{SF} / \mathrm{BIP}^{\text {Baseline * }} 100}
$$

mit j $=2007,2008, \ldots, 2020$.
Tabelle 3

Wirkungen der EU-Strukturfonds 2007-2013: Kumulierter SF-Multiplikator im Jahr 2020

\begin{tabular}{|c|c|}
\hline Tschechische Republik & 2,8 \\
\hline Slowenien & 2,5 \\
\hline Estland & 2,4 \\
\hline Polen & 2,4 \\
\hline Portugal & 2,0 \\
\hline Lettland & 1,8 \\
\hline Rumänien & 1,8 \\
\hline Spanien & 1,7 \\
\hline Ungarn & 1,7 \\
\hline Ostdeutschland & 1,2 \\
\hline Italiens Mezzogiorno & 1,1 \\
\hline Griechenland & 0,9 \\
\hline
\end{tabular}

Quelle: Bradley et al. (2004, S. 57 ff.)

Tabelle 3 zeigt die Ergebnisse für die untersuchten Förderregionen zum Zeitpunkt 2020. Die Spannbreite des kumulierten SF-Multiplikators reicht von 2,8 für die Tschechische Republik bis 0,9 für Griechenland. Die Unterschiede ergeben sich ausschließlich aufgrund der Struktur der betrachteten Volkswirtschaften, so dass sich deutliche Unterschiede hinsichtlich der Effektivität der EU-Strukturfonds-interventionen ergeben. Die Spilloverelastizitäten sind für alle als identisch angenommen, und die Aufteilung der Strukturfonds auf die ökonomischen Kategorien Infrastrukturausbau, Förderung des Humankapitals und der privaten Investitionen sind nicht wesentlich unterschiedlich.

Bevor auf die Ergebnisse aller Länder nochmals eingegangen wird, soll zunächst für die beiden Empfängerregionen Ostdeutschland und die Tschechische Republik die Entwicklung der Wirkungen der Strukturfondsinterventionen näher betrachtet werden. Sie wurden hier gewählt, da die beiden kumulierten Multiplikatoren sich deutlich unterscheiden und für die Tschechische Republik ein Wert von 2,8 und für Ostdeutschland ein Wert von 1,2 und damit ein deutlich unterschiedlicher Impact von den EU-Strukturfonds angezeigt wird. Abbildung 4 zeigt für die beiden Regionen die Entwicklung hin zu den unterschiedlichen Multiplikatorwerten im Jahr 2020. Abbildung 4a zeigt den jährlichen Impuls, der über die Strukturfonds auf die Volkswirtschaften wirkt und die entsprechende Wirkung auf das BIP. Die Implementationsphase reicht von 2007 bis 2013, anschließend fällt der Impuls auf den Wert Null zurück, d.h. die Interventionen mit den Europäischen Strukturfonds enden 2013 und es wird kein irgendwie aus- 
Abbildung 4

Wirkungen der Strukturfonds in der Tschechischen Republik und Ostdeutschland 2007-2013, modelliert bis zum Jahr 2020

a) Jährlicher Impuls der EU-Strukturfonds (CSF) und BIP-Response (GDP)

Ostdeutschland

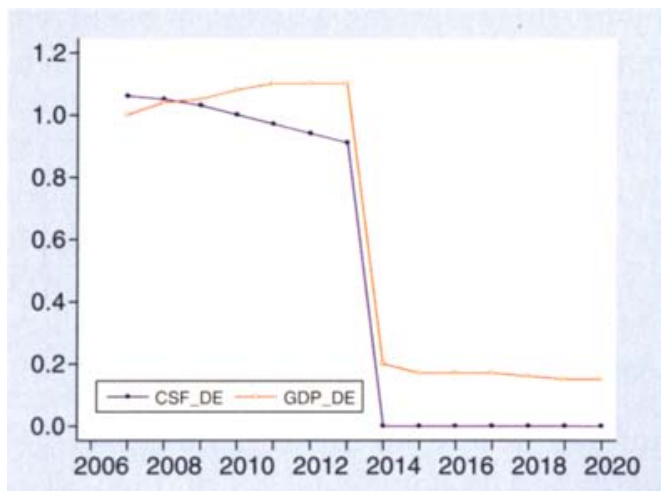

Tschechische Republik

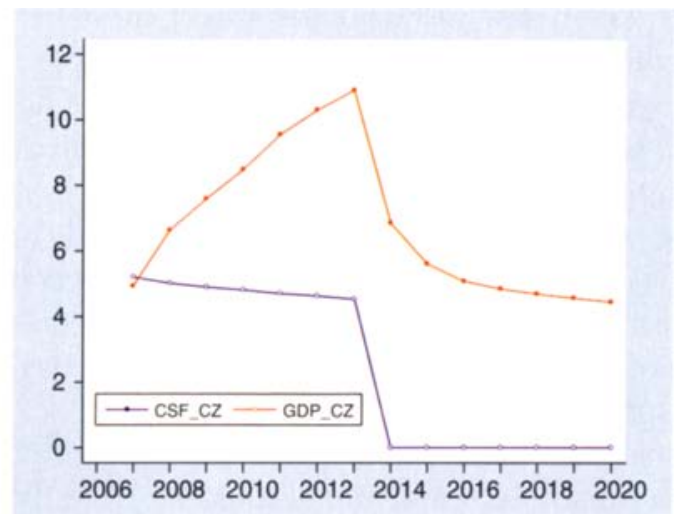

b) Kumulierter Impuls der EU-Strukturfonds (CUMCSF) und kumulierter BIP-Response (CUMGDP)

Ostdeutschland

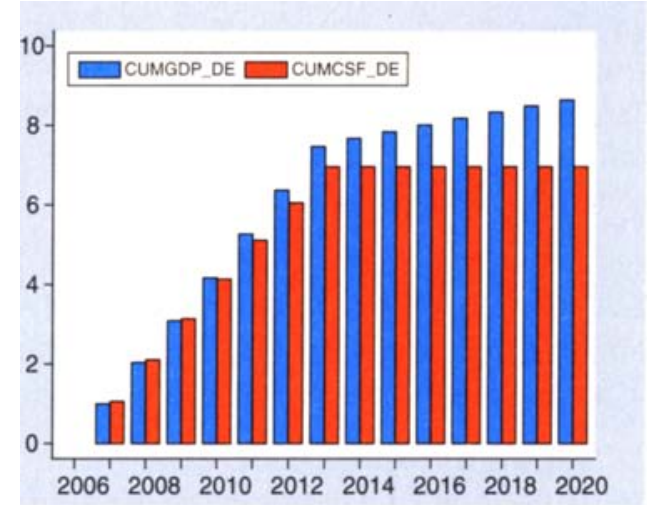

c) Kumulierter SF-Multiplikator (siehe Gleichung oben) Ostdeutschland

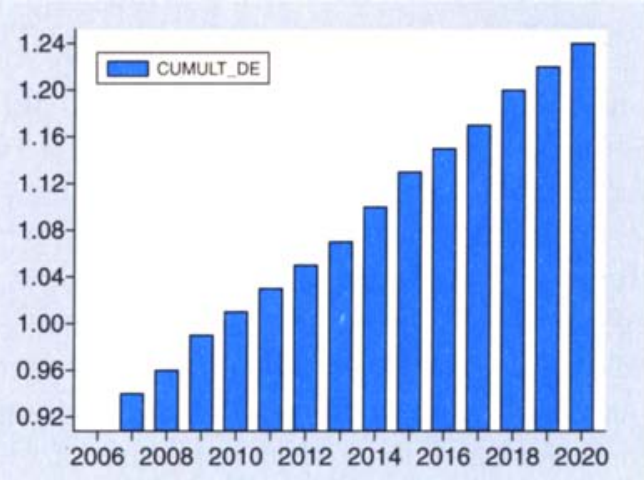

Quelle: Bradley et al. (2004)
Tschechische Republik

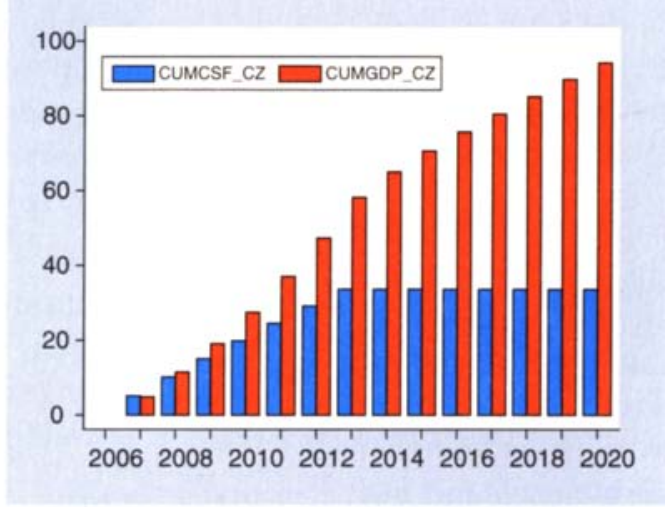

Tschechische Republik

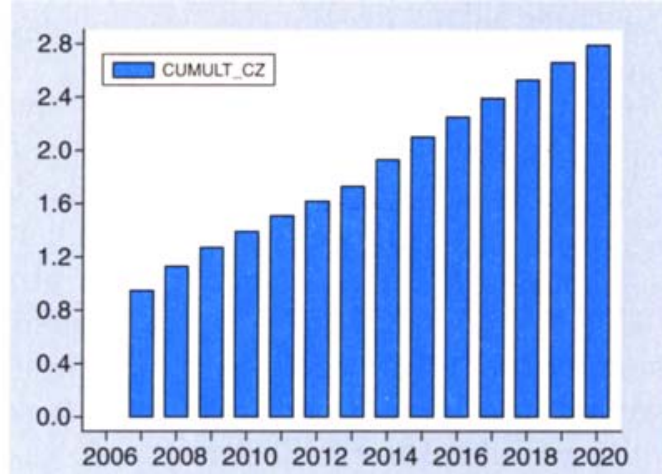


gestaltetes Nachfolgeprogramm aufgelegt, unabhängig davon, ob national oder EU finanziert. Der jährliche Impuls während der Implementationsphase in Ostdeutschland liegt bei etwa $1 \%$ des regionalen BIP und der für die Tschechische Republik bei ungefähr $5 \%$ des tschechischen BIP. ${ }^{6}$ Der Anteil der EU-Strukturfonds am BIP nimmt aber tendenziell ab, da die finanziellen Mittel in sieben gleich große Jahresscheiben auf die Implementationsphase verteilt wurden und die Volkswirtschaften auch in der Baseline eine positive wirtschaftliche Entwicklung aufweisen. Zunächst ist die jährliche Wirkung auf das BIP kleiner als der Impuls. Der Einfluss der Strukturfondsinterventionen nimmt im Zeitablauf zu, wobei der keynes'sche Multiplikator in der Implementationsphase die Entwicklung dominiert und die angebotsseitigen Effekte erst langsam an Einfluss gewinnen. Nach Ablauf der Implementationsphase fällt der positive Impuls ab dem Jahre 2014 auf Null zurück und es verbleiben lediglich die Wirkungen, die auf die Verbesserung der angebotsseitigen Struktur zurückzuführen sind. Mit Blick auf Ostdeutschland beträgt die angebotsseitige Wirkung etwa $20 \%$ der BIPWirkung der Implementationsphase, während der Wert für die Tschechische Republik bei ungefähr $50 \%$ liegt.

Abbildung 4b zeigt nun die Entwicklung des kumulierten prozentualen Anstiegs des Niveaus des BIP für beide Volkswirtschaften, die durch die EU-Strukturfondsinterventionen in Prozent des BIP ausgelöst werden. Zunächst liegt der kumulierte BIP-Einfluss unterhalb des kumulierten Finanzmitteleinsatzes der EU-Strukturfonds. Für Ostdeutschland bleibt ab dem Jahr 2014 der kumulierte Einfluss der EU-Strukturfonds auf einem Niveau von knapp $7 \%$, während es weiterhin positive BIP-Effekte gibt, die am Ende des $\mathrm{Si}$ mulationszeitraums im Jahr 2020 knapp $9 \%$ betragen. Für die Tschechische Republik zeigt sich ein ähnliches Verlaufsmuster, wenn auch auf einem anderen Niveau, da die Interventionen mit den EU-Strukturfonds fünfmal so hoch sind wie in Ostdeutschland. Der Einsatz der EU-Strukturfonds liegt bei ca. $30 \%$ und die kumulierte BIP-Wirkung bei mehr als $80 \%$.

Die Entwicklung des kumulierten SF-Multiplikators zeigt Abbildung 4c. Ausgehend von einem Wert, der knapp unterhalb von Eins liegt, steigt dieser in beiden Fällen im Zeitablauf an und liegt 2020 für Ostdeutschland bei 1,2 und für die Tschechische Republik bei 2,8. Bei der Interpretation der kumulierten Multiplikatoren sollten drei Sachverhalte beachtet werden: Erstens wurden für Ostdeutschland und die Tschechische Republik (wie auch für alle übrigen Empfängerländer) identische Spilloverelastizitäten unterstellt, so dass Unterschiede in der Effektivität des Einsatzes der EUStrukturfonds über die Modellstruktur und die Para- metrisierung determiniert werden, die als Abbild der Struktur der untersuchten Volkswirtschaft betrachtet werden kann. Zweitens wurden aus der internationalen Literatur Spilloverelastizitäten verwendet, die im mittleren Bereich liegen. Höhere Elastizitäten würden höhere kumulierte Multiplikatoren ausweisen, niedrigere zu geringeren kumulierten Multiplikatoren führen. Drittens wurde unterstellt, dass die Kapitalbestände an physischer Infrastruktur und Humankapital mit 2,5\% bzw. $5 \%$ jährlich abgeschrieben werden. Diese liegen möglicherweise im unteren Bereich. Höhere Abschreibungsraten würden zu einer schnelleren Abnahme der angebotsseitigen Wirkungen führen, allerdings würde sich das gezeigte Bild nur geringfügig ändern.

Mit Blick auf Tabelle 3 legen die Ergebnisse der gesamtwirtschaftlichen Wirkungsanalyse für den Zeitraum 2007-2013 nahe, die Empfängerländer in drei Gruppen hinsichtlich der Effektivität des Einsatzes der EU-Strukturfonds einzuteilen:

- Eine erste Gruppe mit kumulierten SF-Multiplikatoren zwischen 2,8 und 2,0. In diese Gruppe fallen die Tschechische Republik, Slowenien, Estland, Polen und Portugal. Für diese Staaten sind die Returns aus den EU-Strukturfonds hoch.

- Eine zweite Gruppe mit kumulierten SF-Multiplikatoren zwischen 1,6 und 2,0. Sie umfasst Lettland, Rumänien, Spanien und Ungarn. Der „Return on Invest" kann hier als durchschnittlich bezeichnet werden.

- Eine dritte Gruppe mit kumulierten SF-Multiplikatoren, die um den Wert 1 schwanken. Diese Gruppe umfasst Ostdeutschland, Italiens Mezzogiorno und Griechenland. In diesen Regionen sind die Rückflüsse der EU-Strukturfondsinvestitionen niedrig.

Es sei nochmals darauf hingewiesen, dass in allen Modellsimulationen ein identisches Set von Parametern für die Spillovereffekte verwendet wurde. Unterschiede in der Performance der EU-Strukturfonds müssen in den Strukturen der zugrunde liegenden Volkswirtschaften liegen. Da mit Ausnahme von Griechenland, Portugal und Spanien nur Regionen aus dem früheren Ostblock einschließlich der DDR in die Untersuchung eingegangen sind, muss auf die vergleichsweise kurzen Beobachtungszeiträume verwiesen werden, die eine makroökonomische Modellierung erschweren. Die Ergebnisse sollten damit mit einiger Vorsicht hinsichtlich der exakten numerischen Werte interpretiert werden. Allerdings zeigen sich deutliche Unterschiede in der Effizienz, die den Schluss nahelegen, dass eine Konzentration der EU-Finanzmittel auf die Länder mit hohen kumulierten SF-Multiplikatoren die Effizienz der EU-Strukturfonds verbessern würde. 


\section{Zusammenfassung und Schlussfolgerungen}

Nach diesem kurzen Überblick über die potenziellen Wirkungen der EU-Strukturfondsförderung im Zeitraum 2007-2013 soll im Folgenden eine kurze qualitative Zusammenfassung einiger Ergebnisse aus dem Einsatz von HERMIN-Modellen zur Messung der Wirkungen der EU-Strukturfonds erfolgen.

Erstens: Bei Betrachtung der reinen Keynesschen Effekte der Strukturfonds, unter der Annahme, dass die angebotsseitigen Wirkungen gleich Null sind, entsprechen die Wirkungen denjenigen anderer makroökonometrischer Modelle. Sie liegen im Bereich zwischen 1,0 für Irland und Estland und 1,5 für Portugal und Spanien. Wenn die Parameter der Externalitäten gleich Null gesetzt werden, haben die EU-Strukturfonds in der langen Frist keinen Einfluss auf die wirtschaftliche Entwicklung der unterstützten Region.

Zweitens: Höhere Werte für die Externalitäten lassen die Wirkungen der EU-Strukturfonds auf die unterstützte Wirtschaft außerhalb der Implementationsphase ansteigen. Insgesamt ist es aber sehr schwierig, exakte Werte für die Elastizitäten zu ermitteln; die internationale Literatur weist aber im Durchschnitt positive Werte aus. Schlecht konzipierte EU-Strukturfondsprogramme, die entweder aus ungeeigneten Maßnahmen bestehen oder nicht hinreichend Bezug auf die Situation des zu unterstützenden Raums nehmen, werden lediglich geringe oder keine langfristigen Wirkungen entfalten. Niedrige Spillovereffekte bringen dies zum Ausdruck. Für die Erstellung der Operationellen Programme bedeutet dies, dass eigentlich ausschließlich auf die langfristigen Implikationen der Maßnahmen zu achten ist. Kurzfristig führt jeder finanzielle Impuls staatlicher Interventionen zu einer Ausweitung der gesamtwirtschaftlichen Nachfrage, sofern kein vollständiges Crowding-out angenommen werden kann. Maßnahmen, die den notwendigen Anforderungen nicht gerecht werden, führen nicht zu einer Veränderung der angebotsseitigen Elemente, und nach Ende der Intervention ist die Situation in der betrachteten Volkswirtschaft unverändert.

Drittens: Selbst wenn die Maßnahmen geeignet und angemessen bezüglich der Ausgangssituation sind, ist damit nicht garantiert, dass sich auch die erhofften Wirkungen einstellen. Sind die Rahmenbedingungen für die makro- und mikroökonomischen Politiken nicht stimmig, können die potenziell positiven Wirkungen versickern. Es scheint der Fall zu sein, dass weniger stark regulierte Volkswirtschaften bei gleichen Spilloverelastizitäten höhere langfristige Returns erbringen.

Viertens: Die transitorischen Interventionen mit den EU-Strukturfonds führen auch langfristig nicht zu einer
Veränderung der Wachstumsrate des Sozialprodukts. Stattdessen kommt es zu einem positiven Niveaueffekt auf das BIP.

Die Analyse der Wirkungen der EU-Strukturfonds in der letzten Dekade hat gezeigt, dass die makroökonomische Modellierung im Prinzip in der Lage ist, die (isolierten) Effekte der Strukturfonds auf die unterstützten Volkeswirtschaften zu zeigen, und dies vor dem Hintergrund nationaler und internationaler Schocks, die auf die Volkswirtschaft wirken. Aber makroökonomische Evaluationen leiden unter dem „Blackbox-Problem“, wobei durch die Modellentwickler an die politischen Akteure Antworten gegeben werden, die durch eine Menge von spezifischen Annahmen und Einschränkungen mitbestimmt sind. Die Vorteile eines modellbasierten Ansatzes liegen darin, dass ein konsistenter analytischer Rahmen genutzt wird, um die untersuchte Volkswirtschaft zu repräsentieren, und dass die Möglichkeit besteht, quantitative Feedback- und Politikbezüge abzubilden und die Wirkungen unterschiedlicher Politiken auf die Entwicklung der Einkommen pro Kopf unter bestimmten Annahmen zu zeigen.

\section{Anmerkungen}

(1)

Während der Laufzeit der europäischen Strukturfondsförderung hat sich die Gebietskulisse mehrfach geändert. In der ersten Förderperioden von 1988 bis 1999 standen die sogenannten Kohäsionsländer im Vordergrund: Irland, Portugal, Griechenland, Nordirland, (weite Teile) Spaniens und das italienische Mezzogiorno. De facto gehörte auch Ostdeutschland ab $1992 \mathrm{zu}$ den Ziel-1-Regionen. Diese Gebietskulisse blieb bis 2004 mehr oder weniger konstant. Mit dem Beitritt der neuen Mitgliedstaaten aus Mittel- und Osteuropa zum 1. Mai 2004 und zum 1. Januar 2007 hat sich dieses signifikant geändert. Alle neuen Mitgliedstaaten fallen unter die Konvergenzregionen, eine Ausnahme ist die Region um Prag, und der Schwerpunkt der Interventionen hat sich deutlich nach Mittel- und Osteuropa verlagert.

(2)

Vgl. European Commission (2006)

(3)

Vgl. Rodrik (2005)

(4)

Die HERMIN-Modelle zeichnen sich dadurch aus, dass sie speziell zur Analyse der EU-Strukturfonds entwickelt worden sind. Sie weisen damit andere Charakteristika auf als jene Modelle, die primär für andere Zwecke entwickelt wurden, wie z.B. das QUEST-Modell. Daher beschränkt sich die Darstellung auf diesen von den Autoren vertretenen Modellrahmen. Eine detaillierte formale Darstellung, wie die Interventionen der EU-Strukturfonds die Entwicklung der unterstützen Volkswirtschaft beeinflussen, ist in Bradley/Gacs/Morgenroth/Untiedt (2004; http://ec.europa. eu/regional_policy/sources/docgener/studies/study_de.htm) enthalten. 
(5)

Ein Beispiel für fixierte Modellstrukturen soll am Beispiel der Produktionsfunktion verdeutlicht werden: Dabei können „exogene“ Entwicklungen in der Form dargestellt werden, dass Änderungen in Variablen (z. B. die relativen Faktorpreise) zu Änderungen in anderen Variablen (z. B. das Faktoreinsatzverhältnis) führen, ohne die Form der Produktionsfunktion zu verändern.

(6)

Das finanzielle Volumen der Interventionen mit den europäischen Strukturfonds besteht aus den Finanzmitteln der EU und der nationalen Kofinanzierung. Private Kofinanzierungen werden nicht berücksichtigt.

\section{Literatur}

Aghion, P.; Howitt, P.:Appropriate Growth Policy: A Unifying Framework (2005; www.economics.harvard.edu/faculty/aghion/papers.html)

Beutel, J.: The Economic Impacts of the Community Support Frameworks of the Objective 1 Regions 1989-1993. Report to DG for Regional Policies. - Brussels 1993

Beutel, J.: The economic impact of Objective 1 interventions for the period 2000-2006. Report to EU DG XVI. - Brussels 2002 (www.dgfc.sgpg.meh.es/aplweb/pdf/DescargasFondosComunitarios/ImpactoFFEEObj1 Beutel2000_06.pdf)

Bradley, J.; Whelan, K.; Wright, J.: The HERMIN project. Economic Modelling 12 (1995) 3, S. 249-274

Bradley, J.; Morgenroth, E.; Untiedt, G.: Macro-regional evaluation of the Structural Funds using the HERMIN modelling framework. Scienze regionali/Italian Journ. of Regional Science (2003) 3

Bradley, J.; Gács, J.; Morgenroth, E.; Untiedt, G.: A Study of the Macro-economic Impact of the Reform of EU Cohesion Policy. Report submitted to the European Commission, Directorate-General for Regional Policy. - Brussels 2004 (http://europa.eu.int/ comm/regional_policy/sources/docgener/studies/pdf/3cr/ macro_impact.pdf)

Bradley, J.; Mitze, T.; Morgenroth, E.; Untiedt, G.: How can we know if EU Cohesion Policy is successful? Integrating micro and macro approaches to the evaluation of Structural Funds. - Münster 2006. = GEFRA Working Paper (www.gefra-muenster.org/ deutsch/publikationen/abstract.php?pub_id=96)

Bradley, J.; Untiedt, G.: The COHESION system of HERMIN country and regional models: Description and operating manual. Draft Report for DG Regional Policy. - Brussels 2007 (a)

Bradley, J.; Untiedt, G.: Do economic models tell us anything useful about Cohesion Policy impacts? - Münster 2007 (b) . = GEFRA Working Paper No. 3 (www.gefra-muenster.org/index.php\#)

Bradley, J.; Untiedt G.; Mitze T.: Analysis of the Impact of Cohesion Policy. A note explaining the HERMIN-based simulations. Draft Report for DG Regional Policy. - Brussels 2007 (c)

Congressional Budget Office: R\&D and Productivity Growth. Congressional Budget Office, The Congress of the United States, June 2005 de la Fuente, A.: Human capital in growth regressions: how much difference does data quality make? Journ. of the European Economic association 4 (2006) 1, S. 1-36

Ederveen, S.; de Groot, H.; Nahuis, R.: Fertile soil for structural funds?: A panel data analysis of the conditional effectiveness of European cohesion policy. --The Hague: CPB 2002 (also available as discussion paper of the Tinbergen Institute: www.tinbergen. $\mathrm{nl} /$ discussionpapers/02096.pdf)

Eggert, W., Ehrlich, M. v.; Fenge, R.; König, G.: Konvergenz- und Wachstumseffekte der europäischen Regionalpolitik in Deutschland. Perspektiven der Wirtschaftspolitik 8 (2007) 2, S. 130-46

European Commission: Annex to the Fourth progress report on Cohesion: Growth and jobs and the Reform of European cohesion policy. COM(2006) 281 final, June 2006

Fan, W.; Treyz, F; Treyz, G.: An evolutionary new economic geography model. Journ. of Regional Science 40 (2000) 4, S. 671-695

Honohan, P.: EU structural funds in Ireland: a mid-term evaluation of the CSF 1994-99. - Dublin: ESRI 1997. = Policy research series 31

Rodrik, D.: Why we learn nothing from regressing Economic Growth on Policies (2005; http://ksghome.harvard.edu/ drodrik/ papers.html)

Roeger, W. ; in't Veld, J. :QUEST II. A Multy Country Business Cycle and Growth Model. - Brussels 1996. = Economic Papers from Commission of the EC No. 123

Tinbergen, J.: A method and its application to investment activity. - Geneva: League of Nations 1939. = Statistical Testing of Business-Cycle Theories 1

Tondl, G.: What determined the uneven growth of Europe's Southern regions?: An empirical study with panel data. - Wien 1999. = IEF working paper 30

Treyz, G.: Regional economic modeling: a systematic approach to economic forecasting and policy analysis. - Boston 1993. $=$ Kluwer Acad. Publ.

Dr. John Bradley

EMDS - Economic Modelling and Development Systems

14 Bloomfield Avenue

Dublin 8

Ireland

E-Mail: john.bradley@iol.ie

Dr. Gerhard Untiedt

GEFRA - Gesellschaft für Finanz- und Regionalanalysen

Ludgeristraße 56

48143 Münster

Germany

E-Mail: untiedt@gefra-muenster.de 